\title{
Reversions to consensus are positively selected in HIV-1 and bias substitution rate estimates
}

\author{
Valentin Druelle ${ }^{1,2}$ and Richard A. Neher ${ }^{1,2}$ \\ ${ }^{1}$ Biozentrum, University of Basel, Basel, Switzerland \\ ${ }^{2}$ Swiss Institute of Bioinformatics, Basel, Switzerland
}

February 13, 2022

\begin{abstract}
HIV-1 is a rapidly evolving virus able to evade host immunity through rapid adaptation during chronic infection. The HIV-1 group M has diversified since its zoonosis into several subtypes at a rate of the order of $10^{-3}$ changes per site per year. This rate varies between different parts of the genome and its inference is sensitive to the time scale and diversity spanned by the sequence data used. Higher rates are estimated on short time scales and particularly for within-host evolution, while rate estimates spanning decades or the entire HIV-1 pandemic tend to be lower. The underlying causes of this difference are not well understood.

We investigate here the role of rapid reversions toward a preferred evolutionary sequence state on multiple time scales. We show that within-host reversion mutations are under positive selection and contribute substantially to sequence turnover, especially at conserved sites. We then use the rates of reversions and non-reversions estimated from longitudinal within-host data to parametrize a phylogenetic sequence evolution model. Sequence simulation of this model on HIV-1 phylogenies reproduces diversity and apparent evolutionary rates of HIV-1 in gag and pol, suggesting that a tendency to rapidly revert to a consensus-like state can explain much of the time dependence of evolutionary rate estimates in HIV-1.
\end{abstract}

\section{Introduction}

RNA viruses have low fidelity polymerases, resulting in a rapidly diversifying virus population, which, in turn, facilitates the adaptation to changing environments. The human immunodeficiency virus 1 (HIV-1) is a prime example of such a rapidly evolving virus. The life-long infections it causes are characterized by a large viral population that accumulates diversity at a high rate to constantly evade host immunity (Coffin, Swanstrom, 2013). This continuous evolution has led to a diverse viral population on the pandemic scale that is categorized into several viral subtypes (Brian Foley, 2018; Li et al., 2015). Different lineages have accumulated diversity at a rate of about 1 substitution in 1000 sites per year since its jump to human hosts at the turn of the 20th century (McCutchan, 2006, Sharp, Hahn, 2011; Korber et al. 2000).

Quantifying the rate of viral evolution, however, is surprisingly difficult and different approaches yield different answers. Most importantly, the time scale across which sequences are compared affects the estimates strongly, sometimes by orders of magnitude: the longer the time scale, the lower the estimate (Aiewsakun, Katzourakis, 2016, Hanada et al., 2004, Worobey et al., 2010, Gilbert, Feschotte, 2010, Ghafari et al., 2021). These discrepancies suggest that we lack a good understanding of how micro-evolutionary within-host (WH) processes on the scales of days, months and years give rise to the diversity observed on longer time scales across hosts. In the case of chronic infections such as HIV-1, these micro-evolutionary processes are driven by selection to evade a changing host immune response at each transmission while maintaining fitness.

HIV-1 is an ideal system to study these effects in detail since the rate discrepancies between the WH, pandemic and broader scales are well documented (Alizon, Fraser, 2013, Worobey et al., 2010), the pandemic is well sampled, and high resolution WH data exists. The evolutionary rate estimated on the pandemic scale is around two to five times lower than the one observed within host (Alizon, Fraser, 2013). Several hypotheses have been put forward to explain this phenomenon. Two of the main hypotheses are the preferential transmission of ancestral HIV-1 variants, i.e. the "store and retrieve" hypothesis (Lythgoe, Fraser, 2012), 
and the reversion toward an ancestral-like state, i.e. the "adapt and revert" hypothesis (Redd et al., 2012 Zanini et al., 2015: Leslie et al., 2004). The relative importance of these and possibly other processes for the discrepancy of rate estimates is not well understood (Raghwani et al., 2018).

We use WH longitudinal deep sequencing data to investigate the evolutionary processes responsible for the difference of evolution rate observed. We, firstly, show that rate estimates are related to the time scale and reference point used on both the $\mathrm{WH}$ and pandemic scale, suggesting a saturation of divergence long before it is expected in most sequence evolution models. Secondly, we investigate the cause of this saturation and find that reversion towards the HIV-1 consensus is more common than expected. Such WH reversion mutations are positively selected. Lastly, we use simulations of evolution to quantify the impact of such reversions on rate estimates on time scales of decades and above. More generally, our results highlight the evolutionary bias of viruses toward a consensus or state of high intrinsic fitness in a changing environment.

\section{Results}

We use a (i) a set of sequences representative of the HIV-1 pandemic spanning multiple decades and a (ii) longitudinal data set following the evolution of the virus within individual hosts to investigate patterns of evolution on multiple time scales. The former between-host (BH) data set contains 1000 HIV-1 group M sequences from the Los Alamos National Laboratory HIV database (Foley et al., 2013). Subsampling was performed to have the same number of sequences for each year to avoid sampling biases (except for early years, where fewer sequences are available) but otherwise randomly picked from the full data set. Our WH analysis is based on the HIVEVO data set (Zanini et al. 2015), a whole genome deep sequencing of HIV-1 populations in 11 patients during a 4 to 16 years follow-up without treatment. Between 6 and 12 samples are available per patient. Sequencing depth and template input of all samples in this data set has been assessed and most samples allow a confident calling of frequencies of minor variation down to a few percent (Zanini et al. 2016). See section M\&M 1.1 for details.

We analyze the evolution of the env, pol and gag genes of HIV-1 below. They code for surface proteins, viral enzymes and capsid proteins, respectively (Freed, 2001). When combined, they cover approximately $80 \%$ of the genome. We focus on the pol region in the main text and present analogous results for the env and gag regions in the Supplementary Materials.

\section{Apparent rates depend strongly on the distance to the reference sequences}

The rate at which differences between sequences accumulate decreases with time as more and more sites are hit multiple times by changes (Felsenstein, 2004). If all sites evolve at the same speed, such saturation effects are only important once distances between sequences are large (the size of correction is proportional to the distance squared). However, if different sites evolve at drastically different rates, or reversions are common, such saturation effects set in much earlier and can lead to significant deviations even when sequences are still very similar (Puller et al., 2020, Ghafari et al., 2021).

Figure 1 compares the rate of accumulation of sequence changes of HIV-1 at the pandemic scale (A) and within hosts (B) in the pol gene. Figure 1 A shows the Hamming distance of HIV-1 sequences from the inferred root of the HIV-1 group M tree (orange) or the consensus of the subtype (green). These Hamming distances are compared to the corresponding increase in root-to-tip (RTT) differences along a reconstructed phylogeny (blue).

The slope of the Hamming distance relative to the subtype consensus is larger than that relative to the root, while the slope of the RTT regression is even larger. These substantial differences suggest that saturation effects are already strong despite the fact that distances are less than $10 \%$.

The Hamming distance to subtype consensi (green) was computed for HIV-1 subtype B and C sequences, relative to their respective subtype consensus computed from the alignment. The phylogenetic tree was inferred using an IQTree GTR+F+R10 model (Tavaré, others, 1986; Yang, 1995; Minh et al., 2020), which was found to be the best model according to the IQTree model finder (Kalyaanamoorthy et al., 2017). For more details, see section $\mathrm{M} \& \mathrm{M} 1.2$.

Such rapid saturation can arise through rate variation (Soubrier et al., 2012) or heavily skewed site-specific equilibrium frequencies resulting in rapid reversion (Halpern, Bruno, 1998, Hilton, Bloom, 2018, Puller et al. 2020 Ho et al., 2005, Wertheim, Kosakovsky Pond 2011). We perform a similar analysis on WH data on time scales of 3-10 years to differentiate between the different explanations of rate variation and reversion. 

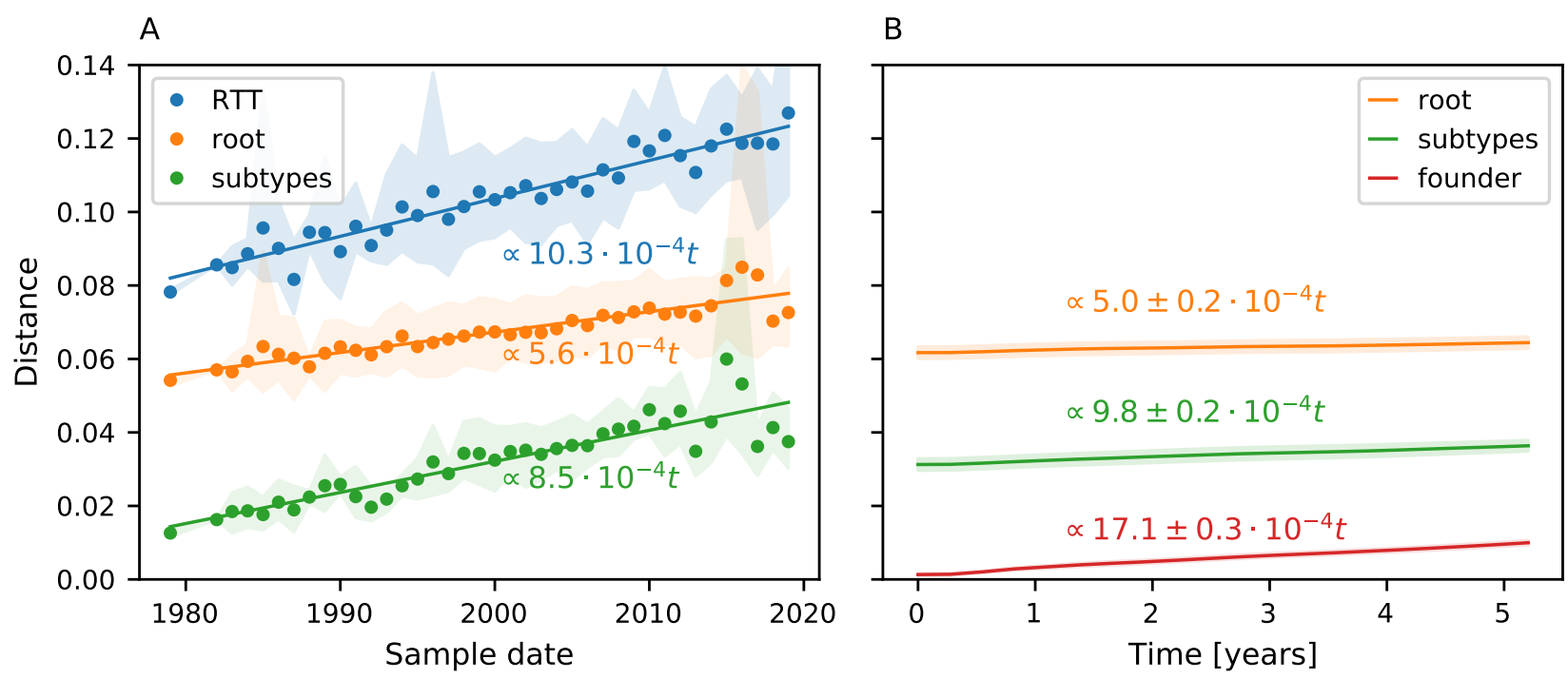

Figure 1: Divergence over time in the pol gene. A: The graph shows the average Hamming distance from the root of the HIV-1 group M tree, from the respective subtype, or RTT distance as a function of time. Each data point is the average of sequences from one year; the shaded area indicates the $10-90 \%$ range. B: The WH divergence over time relative to the putative founder genotype and HIV-1 group M root or subtype consensi, averaged over all patients in the HIVEVO data set. Divergence is computed according to equations 1 and 2. The Hamming distance to the HIV-1 group M root or the subtype consensi increases at similar rates within hosts and cross-sectionally. Results for region env and gag are shown in Supplementary Figures s1 and $: 2$.

We compute WH evolutionary rates by measuring the divergence over time in Figure 2B. Specifically, we calculate the divergence $d(t)$ relative to a reference sequence, such as the root of the tree according to:

$$
d(t)=\frac{1}{N} \sum_{i}^{N} \delta_{i}^{r e f} \cdot\left(1-f_{i}(t)\right)+\left(1-\delta_{i}^{r e f}\right) \cdot f_{i}(t)
$$

where $N$ is the length of the region and $f_{i}(t)$ is the frequency of the founder nucleotide at position $i$ and time $t$ in the viral population. This founder nucleotide is approximated by the majority nucleotide at the first time point and $\delta_{i}^{r e f}$ is a Boolean such that $\delta_{i}^{r e f}=1$ if the founder nucleotide at position $i$ is the same as in the reference sequence and $\delta_{i}^{r e f}=0$ otherwise. More details about the computation of the founder sequence are in section $\mathrm{M} \& \mathrm{M} 1.3$. When measuring the divergence relative to the founder sequence, we instead use the frequencies of the first sample $t=t_{0}$ as follows:

$$
d(t)=\frac{1}{N} \sum_{i}^{N} f_{i}\left(t_{0}\right) \cdot\left(1-f_{i}(t)\right)
$$

Overall the quantity $d(t)$ measures the Hamming distance to the reference sequence expected for a randomly picked virus in the viral population of a sample. We then averaged these quantities over all samples and estimated uncertainty by bootstrapping groups of samples from the same patient with replacement. In analogy to the $\mathrm{BH}$ analysis, we use the root of the HIV-1 group M tree and subtype consensi as reference sequences, supplemented by the founder sequence of each patient. The results are shown in Figure $1 \mathrm{~B}$. The filled areas represent one standard deviation of the bootstrap replicates. For more details about the methodology, see section M\&M 1.2. The divergence increases the fastest relative to the founder sequence at approximately $(17.1 \pm 0.3) \cdot 10^{-4}$ mutations per site per year, 1.7 times the speed observed from the RTT estimate in the BH data set. Saturation effects play a minor role in the WH evolution speed on the time scale of several years. The rate at which the distance to the root or subtype consensus sequence increases, however, is close to the same estimates in the BH data set. Similar results are found for the env and gag regions in Figures $: 1$ and $: 2$ 
A

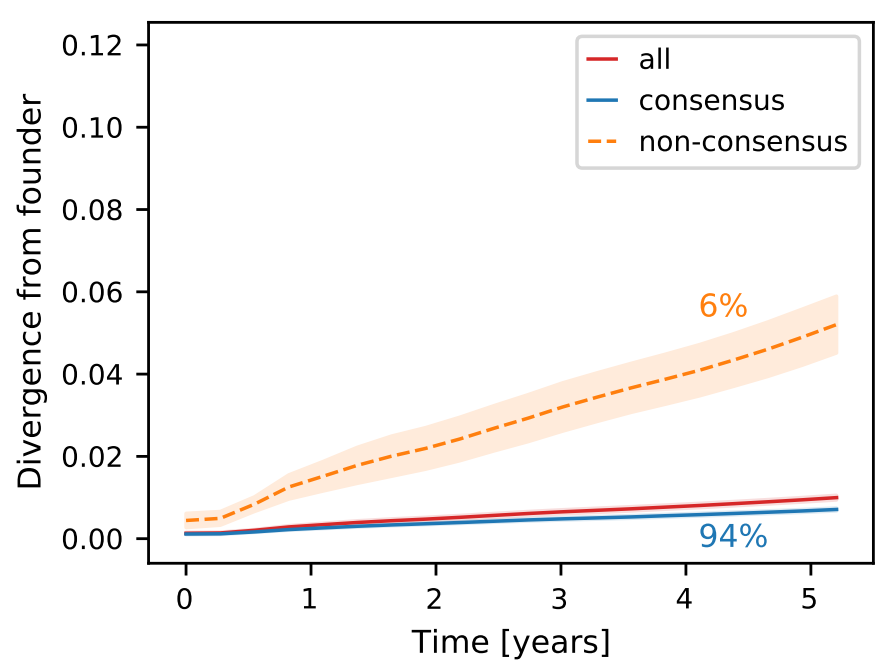

B

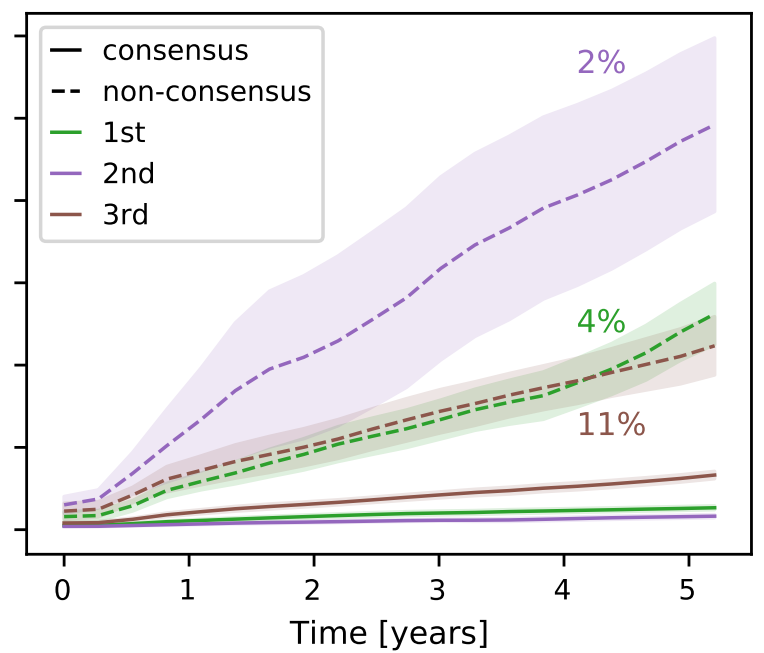

Figure 2: Divergence from founder sequence over time in the pol gene. A: Divergence to founder overall and split for sites initially in consensus and non-consensus states. The reference used to define consensus and non-consensus sites is the HIV-1 group M consensus. Colored percentages are the fraction of sites corresponding to the related curve. Non-consensus sites represent only $6 \%$ of the gene but diverge faster over time. B: The same data as that shown in the left panel split between the 1st, 2nd and 3rd codon position. The difference in evolution speed is greatest for nucleotides in the 2nd position, where mutations can not be synonymous. Results for region env and gag are shown in Supplementary Figures 95 and 94

\section{Non-consensus sites diverge faster}

We next explored the evolution toward and away from consensus within hosts, see Figure 2, Panel 2A shows the WH divergence separately at sites where the founder sequence agrees with the HIV-1 group M consensus and where it differs from it. Filled areas show the standard deviation of the bootstrap estimate. The divergence at sites where the founder sequence differs from the global consensus increases about 7 -fold faster than in the rest of the sequence. In the case of the pol gene, about $6 \%$ of sites fall into the former category, suggesting that about 1 in 3 changes brings the sequence closer to the HIV-1 root sequence. This is consistent with the 3 -fold difference in slope between the divergence relative to the founder or HIV-1 group $\mathrm{M}$ root shown in Figure $1 \mathrm{~B}$.

This accelerated evolution could be due to (i) reversion to an ancestral state to increase fitness, or (ii) reduced purifying selection at sites with high levels of diversity in global HIV-1 population. In order to differentiate between these possibilities, Figure $2 \mathrm{~B}$ shows the divergence by codon position. The degree to which reversions are accelerated differs between the 1st, 2nd and 3rd position in a codon. In particular, sites in the 2nd position diverge the fastest when in a non-consensus state, while they diverge the slowest in a consensus state. This is consistent with a particularly strong pressure to revert at the 2nd position, where mutations cannot be synonymous and tend to correspond to more drastic amino acid changes. Accordingly, non-consensus sites in the 2 nd position are rare as they represent only $2 \%$ of such sites. Similar results are found when using root sequence or subtype consensus sequences as a reference instead. The results for env and $g a g$ are shown in Figures $\mathrm{S} 3$ and $\mathrm{s} 4$. These results are consistent with previous observations showing that conserved sites tend to revert more quickly (Zanini et al. 2015) and the notion that selection for reversion is probably driven by the fitness costs of mutations that enabled immune escape in a previous host (Leslie et al. 2004).

Note that more rapid reversion at conserved sites is inconsistent with the hypothesis that rapid reversion is due to an overall elevated evolutionary rate at sites that are often in a non-consensus state. Conserved sites would typically be assigned to low rate categories in phylogenetic inference, while sites that tend to be assigned to high rate categories are those that are diverse and, in this analysis, show the slowest rate of reversion. 
A

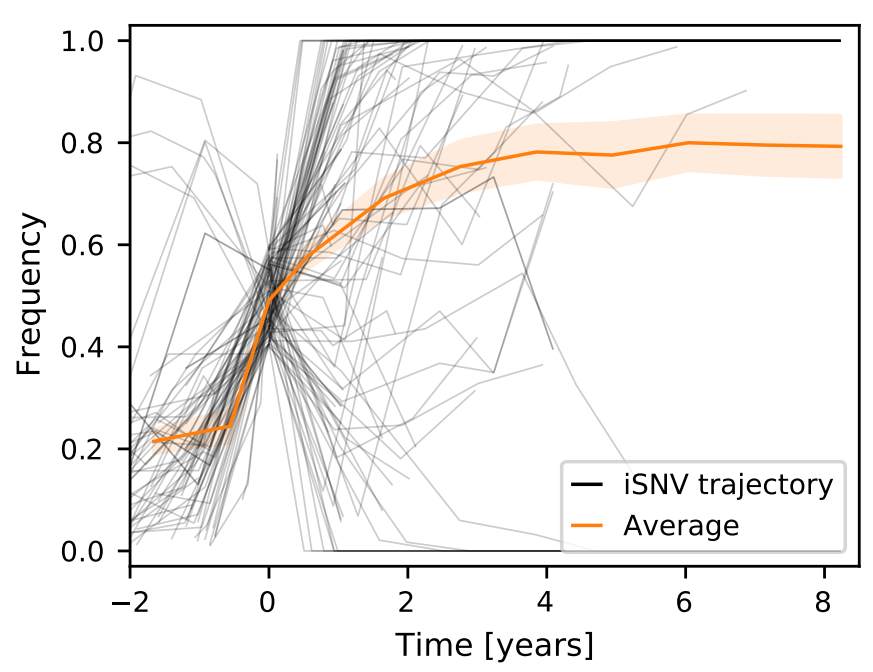

B

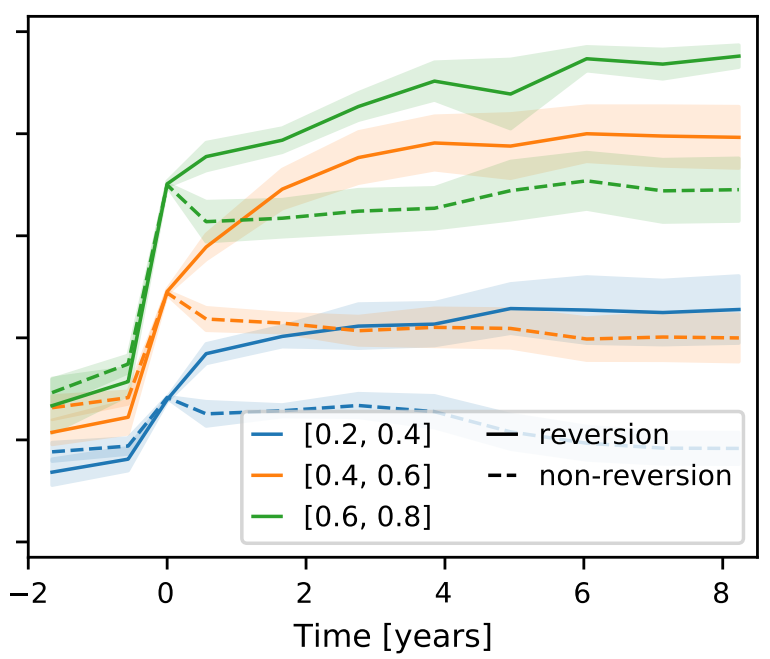

Figure 3: Positive selection on reversion mutations. A: Frequency of reversion mutations seen between 0.4 and 0.6 at one time point (offset to be $t=0$ ) and their average. B: Mean frequency in time for reversion (full lines) and non-reversion mutations (dashed lines) for different frequency windows (colors). Reversion trajectories are strongly selected for as their means increase over time. Non-reversion trajectories evolve close to neutral expectation. The reference sequence used to define reversion mutations is the HIV-1 group M consensus. The solid orange line is the same in both panels.

\section{Reversion mutations are positively selected}

If a lot of reversions are driven by selection, as the codon position-specific analysis above suggests, effects of selection should be detectable in the dynamics of single nucleotide variants within the host (iSNVs). Specifically, we expect reversion mutations to bear signatures of selective sweeps. We analyzed the frequency trajectories of iSNVs to look for such features. Similar to the previous analysis, we separate all trajectories into reversion and non-reversion groups and compare their evolutionary dynamics in Figure 3 We select trajectories with at least one data point in a frequency interval $\left[f_{\min }, f_{\max }\right]$ for each group. We offset these trajectories in time so that $t=0$ corresponds to their first data point in the frequency interval and compute the mean frequency of the trajectory group over time. More details about the definition of trajectories and the methodology are in section M\&M 1.4 and M\&M 1.5. We use the HIV-1 group M consensus sequence as a reference to define reversion mutations, but results are qualitatively similar when using subtype consensus or root sequence as a reference.

Figure 3 A shows individual trajectories shifted to pass through the frequency interval $[0.4,0.6]$ at $t=0$ along with their mean. The mean frequencies for different initial conditions and groups of trajectories are shown in Figure $3 \mathrm{~B}$. Since we condition the set of trajectories to start as minor variants and pass through a frequency interval at $t=0$, we expect that trajectories tend to rise for $t<0$, as is indeed observed. The dynamics at $t>0$, i.e. after the time of conditioning, is informative about the selection of the iSNV. We do not expect any consistent trend to rise or fall in frequency for neutral mutations, and the mean frequency should be flat for $t>0$. Contrary to that, we show in Figure $3 \mathrm{~B}$ that the frequency of reversion mutations increases on average over time. This suggests that these reversion mutations are beneficial on average and fix preferentially in the population, with probability given by the end point of the curves for each group of trajectories. This finding is consistent with the notion that the HIV-1 consensus sequence approximates a fitness optimum of HIV-1 (Zanini et al., 2017). On the other hand, non-reversion curves are flat or slightly decreasing for $t>0$, suggesting that such mutations tend to be slightly selected against or are neutral - at least those that reach high frequency in the first place.

We note that the selection for reversion mutations is strongest for the gag region and weakest for the env region, see Figure $\$ 8$ for details. We also observe a difference in selection for synonymous and non-synonymous mutations (Fig. \$5), consistent with earlier results (Zanini, Neher, 2013). 


\section{Reversions can explain the rate mismatch}

Over longer time scale, the WH reversions we observe will lead to undetected substitutions along branches of the phylogeny whenever a mutation and its corresponding reversion happen on the same branch. When such reversion dynamics are not captured by the substitution models, the evolutionary rate inferred by phylogenetic methods will be too low (Halpern, Bruno, 1998; Hilton, Bloom, 2018, Puller et al., 2020). This bias will contribute to the discrepancy of evolutionary rates within and between hosts.

We quantify the impact of reversion on the $\mathrm{BH}$ evolution rate using a WH informed evolutionary model that accounts for the reversion bias highlighted previously. Firstly, we use the TreeTime library (Sagulenko et al. 2018) to define a site-specific general time reversible (GTR) model to reproduce the evolutionary process (Puller et al. 2020). We parametrize the mutation rate from nucleotide $j$ to $i$ at position $\alpha$ as:

$$
Q_{i j}^{\alpha}=\mu p_{i}^{\alpha} W_{i j}
$$

where $\mu$ is the mean mutation rate per site per year, $p_{i}^{\alpha}$ describes the equilibrium probability of finding nucleotide $i$ at site $\alpha$, and $W_{i j}$ accounts for the overall variation in rate between different nucleotide pairs $i$ and $j$ (e.g. the differences between transitions and transversions). We use $\mu=17.1 \cdot 10^{-4}$, the overall WH evolution rate observed in Figure $1 \mathrm{~B}$. In this model, the bias for reversion is introduced via the equilibrium frequencies $p_{i}^{\alpha}$. These depend on the genome position $\alpha$, enabling us to skew the frequencies towards the consensus nucleotide at each position. Contrary to common evolutionary models that include rate variation between sites, we keep it constant across positions and vary $p_{i}^{\alpha}$ instead. The results are insensitive to Gamma-distributed rate variation with a shape parameter greater than 2 . We choose $p_{i}^{\alpha}$ such that the model reproduces the WH rates of reversions and evolution away from consensus. Specifically, we use

$$
\begin{array}{llr}
p_{i}^{\alpha}=\frac{\mu_{-}^{\alpha}}{\mu_{-}^{\alpha}+\mu_{+}^{\alpha}} & \text { if } & i=\text { consensus } \\
p_{i}^{\alpha}=\frac{\mu_{+}^{\alpha}}{\mu_{-}^{\alpha}+\mu_{+}^{\alpha}} \cdot r_{i}^{\alpha} & \text { if } & i \neq \text { consensus }
\end{array}
$$

where $\mu_{+}^{\alpha}$ and $\mu_{-}^{\alpha}$ are the consensus and non-consensus evolution rate, respectively, computed from results shown in Figure $2 \mathrm{~B}$. These rates are codon position-specific, meaning for every $\alpha$ that is a 1 st codon position $\mu_{+}^{\alpha}=\mu_{+}^{1 s t}$ and $\mu_{-}^{\alpha}=\mu_{-}^{1 s t}$. This is analogous for the 2 nd and $3 \mathrm{rd}$ codon positions. The parameter $r_{i}^{\alpha}$ is used to define the relative proportion of non-consensus nucleotides caused by the uneven transition vs. transversion rates. It is chosen so that $85 \%$ of the non-consensus nucleotides are the transition from the consensus, while the two transversions contribute $7.5 \%$ each. These values were inferred from the $\mathrm{BH}$ alignment. Otherwise, this GTR model is purely informed by WH rates.

We then used this model to simulate evolution along a tree and generate a multiple sequence alignment (MSA). This was done using TreeTime by providing the root sequence and tree inferred from the real BH data, as used in Figure 1. We then inferred a tree from the MSA generated using IQTree, as we did for the real data.

Figure 4 compares the diversity of original and generated MSAs and the length of the inferred trees to quantify the impact of reversions on phylogenetic inference. A model that does not account for reversions, i.e. where $p_{i}=0.25$ for $i \in \mathrm{A}, \mathrm{C}, \mathrm{G}, \mathrm{T}$ for all sites, was included for comparison and is referred to as the naive GTR model. Figures $4 \mathrm{~A}$ and $4 \mathrm{~B}$ show a comparison of the real and generated MSA characteristics. The MSA generated using our WH-informed GTR model (green) has a similar nucleotide content and distance to root as the real BH data (blue). On the contrary, the naive GTR model that does not take reversions into account (orange) results in a more diverse MSA and overall nucleotide content that is less similar to the BH data.

A similar behaviour can be observed from the increase of RTT distances over time, as shown in Figure 4 C. The evolutionary rate estimated from the RTT regression of the naive GTR model is, as expected, very close to the WH evolution rate of $\mu=17.1 \cdot 10^{-4}$ mutation per site per year we input into the model. Our custom GTR model, which uses the same $\mu$ but accounts for reversions, results in a RTT regression with a slope of $13.2 \cdot 10^{-4}$. This suggests that a substitution model parametrized by rates and reversion propensity of $\mathrm{WH}$ evolution can largely reconcile the discrepancy of rate estimates at different scales.

We find qualitatively similar results for the gag and $e n v$ region, see Figures $\$ 6$ and 87 . In the case of env, the tree reconstructed from the data generated and subsequent analysis is unreliable, suggesting that reversions attenuate the clock signal significantly. 

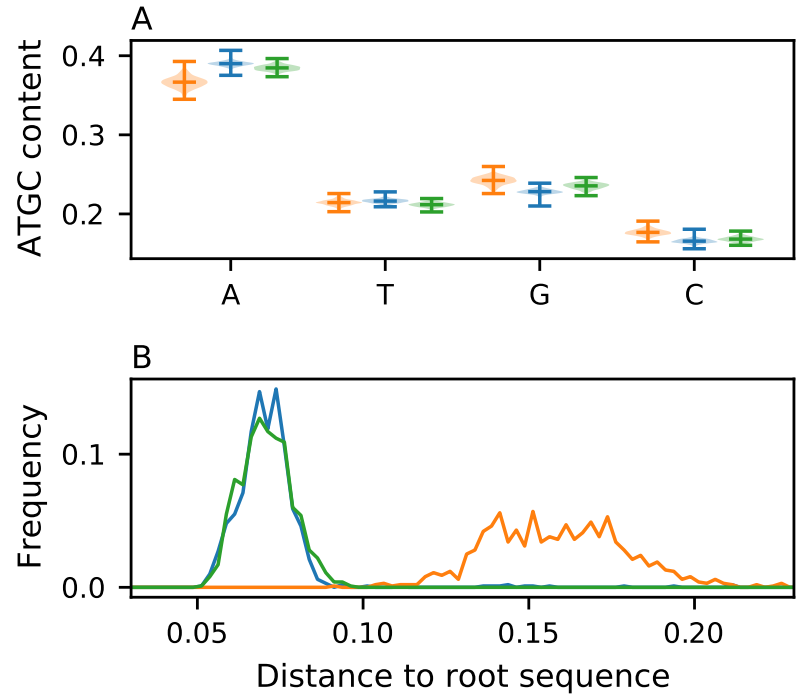

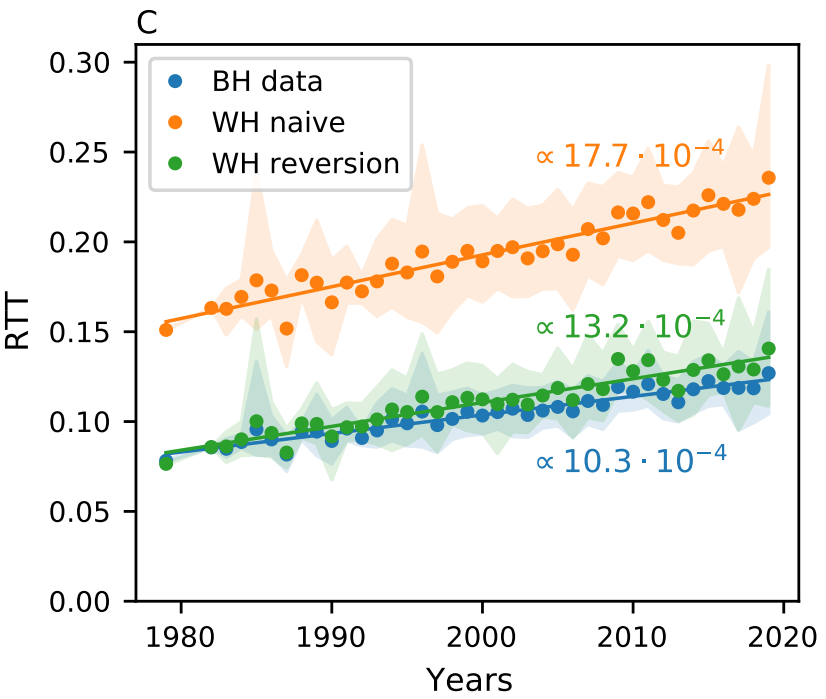

Figure 4: Substitution models that account for reversions can largely explain the rate mismatch. This figure shows the sequence diversity and RTT distances for simulated data generated with a substitution model that account for reversion parametrized by WH data (WH reversion) and a model that does not account for reversion (WH naive) for the pol region. A: Violin plot of the nucleotide content for BH and the MSAs generated. The naive model tends to change the nucleotide content, while the model accounting for reversion matches the $\mathrm{BH}$ observations better. B: Histogram of Hamming distances to the root sequence. The reversion-informed model agrees well with the $\mathrm{BH}$ observations, while the naive one drifts too far from the root. C: While the RTT distance estimated from data generated by the naive model is correctly estimated (true value $17.1 \cdot 10^{-4}$ ), data generated using the model with reversion results in much lower estimates, similar to the rate estimated from $\mathrm{BH}$ data. Results for $e n v$ and $g a g$ are shown in Supplementary Figures $\mathrm{S} 6$ and 97.

\section{Discussion}

Evolutionary rate estimates depend strongly on the time scale over which they are measured (Ho et al. 2005; Aiewsakun, Katzourakis, 2016). Here, we explored this effect on the scale of the HIV-1 pandemic, individual subtypes and within hosts and attempt to bridge these scales. We show that differences between WH estimates and rate estimates on the pandemic scale can, to a substantial degree, be explained by a rapid and strong tendency to revert deleterious mutations to their preferred state. These unpreferred states are probably the result of immune selection in a previous host which gradually revert as the host-specific selection pressure is relaxed in future hosts. Microscopically, we thus observe evolutionary dynamics driven by the adaptation to a changing environment. These transiently beneficial escape mutations are generally deleterious on longer time scales, such that the aggregate effect of this dynamic looks like slowly acting purifying selection (Wertheim, Kosakovsky Pond, 2011).

The rate of reversions observed during chronic infection within hosts can explain approximately $60 \%$ of the apparent slowdown of evolution for the pol gene of HIV-1. A similar selection for reversion mutations has also been observed during acute infection (Boutwell et al., 2010, Leslie et al., 2004) or the transmission bottleneck (Carlson et al. 2014). Such preferential transmission of consensus-like variants will amplify the overall effect of incomplete reversions during chronic infection. Together, these results suggest that, among the hypotheses proposed to explain the difference in rates (Lythgoe, Fraser, 2012, Redd et al., 2012, Zanini et al., 2015, Leslie et al., 2004), the "adapt and revert" mechanism seem to be the main driver of the slowdown.

Substitution models commonly used to reconstruct phylogenies and infer evolutionary rates do not account for rapid reversions, which require site-specific preferences for different states (Halpern, Bruno, 1998; Hilton, Bloom, 2018, Puller et al., 2020). Such model misspecification is particularly problematic on long branches in the phylogenetic tree, where mutations are masked by their corresponding reversions. Such site-specific saturation effects probably affect deep and shallow parts of the tree to a different degree and deep long branches will suffer more severe distortions.

Rapid reversions are probably essential to conserve global fitness by purging costly immune escape mu- 
tations acquired in individuals earlier in the transmission chain (Carlson et al., 2014, Zanini et al., 2017). In addition to reversion, fitness costs of escape mutations can, of course, also be mitigated by compensatory mutations (Crawford et al., 2007, Carlson, Brumme, 2008). Though such compensatory mutations presumably slow down many reversions, we still observe a marked difference in iSNV frequency dynamics towards vs. away from consensus. In addition, compensatory evolution can change the preferred sequence to a new local fitness maximum to which mutations revert, adding an additional slow time scale to the evolutionary process. We expect the preferred sequence to slowly drift on time scales much longer than the typical serial interval. This effect has been observed in deep mutational scanning experiments in influenza viruses (Hilton, Bloom, 2018, Doud et al. 2015).

The star-like diversification of HIV-1 into multiple subtypes gives a clear notion of a consensus sequence that can be used to approximate a putative fitness peak towards which reversions occur. In other viruses, for example, influenza A viruses, the ladder-like or otherwise structured phylogenies do not allow a straightforward definition of a consensus sequence. Nevertheless, it is possible that adaptation to a changing immunity landscape and reversions contribute with a similar magnitude to sequence turnover.

\section{Acknowledgements}

We gratefully acknowledge stimulating discussions with Pierre Barrat-Charlaix and Marco Molari. This work was funded by the core funding of the University of Basel, the SNF (310030_188547) and the PhD Fellowship Program of the Biozentrum (to VD).

\section{Material and Methods}

\subsection{Data set and filtering steps}

\section{Between host data sets}

Our BH data sets come from the Los Alamos National Laboratory HIV databases. All HIV-1 group M sequences containing $0 \%$ problematic nucleotides in the region and an exact sampling date were downloaded for each region. Subtype O and subtype N sequences were filtered out. Only one sequence was kept per patient. Data were downloaded on July 14, 2021. This gave us a total of 6649 sequences for pol, 15034 for env and 8948 for gag.

Regarding each genomic region, we subsampled the data set to have 1000 sequences in each case, with the same number of sequences for each year where sequences were available (except for early years where fewer sequences were available). We then performed an MSA, including the HXB2 sequence, using MAFFT (Katoh, Standley, 2013) and the Nextstrain framework (Huddleston et al., 2021). Insertions relative to the reference HXB2 sequence were removed. We removed all positions of the alignment where more than $10 \%$ sequences have a gap as the alignment can be unreliable in such positions. The alignment for the pol, env and $g a g$ regions are the data sets used for our BH analyses. See section Code and data availability for access to the data sets.

\section{Within-host data sets}

Our WH analysis leverages the time resolution of the HIVEVO data set (Zanini et al., 2015). This data set is freely available with tools made available to facilitate the analysis. We use these tools to obtain a threedimensional matrix of nucleotide frequencies for each patient. The three axes of these tables are the HIV-1 genome position, the nucleotide and the time since infection of the sample. Each entry in these matrices gives the frequency of a given nucleotide at a given position on the genome at this time point, relative to the total intra-patient HIV-1 population. These matrices form our WH data set. We excluded patient $p 7$ and $p 10$ from our analysis as their samples were very uneven in time.

Estimates of nucleotide frequencies are unbiased in the $[0.1,0.9]$ range, while coverage and depth are globally sufficient (Zanini et al. 2016 ). We applied several filtering steps prior to analysis to avoid biases in our results. We, firstly, masked data points with sequencing coverage inferior to 100 and/or where the depth was low. We also removed genome positions that were not mapped to the consensus sequence and/or seen to be too often gapped in the MSA of BH sequences. The alignment and mapping of such sites can be unreliable, thus, we removed them from our analysis. This filtering procedure is mainly relevant for the env gene, which is the region with the most noise. 


\subsection{Distance and divergence over time}

The first result section gives an overview of the method used to compute the distance and divergence over time in Figures 1 , 51 and 52 . Additional details are given below.

Hamming distances were computed by counting the number of sites that do not match the reference sequence for each sequence in the data set. We then divide this number by the length of the sequence to obtain the relative distance to the reference. Hamming distances were computed using three reference sequences. The first is the root sequence of the tree. The tree was inferred using the IQTree GTR $+\mathrm{F}+\mathrm{R} 10$ model (Minh et al., 2020), while the root sequence was computed using TreeTime ancestral reconstruction on this tree (Sagulenko et al. 2018). We chose to use the root sequence instead of the consensus sequence of the alignment in Figures 1 and 4 to avoid biases due to overrepresentation of subgroup B and $\mathrm{C}$ sequences. The second and third reference sequence are subgroup B and subgroup C consensus sequences. See section $\mathrm{M} \& \mathrm{M} 1.3$ for details about the computation of consensus and founder sequences. We performed an average of the distance computed for subtype B and C sequences relative to their consensus to compute the Hamming distances to subtype consensus. The average was weighted by the relative number of each subtype sequence in each year.

The RTT distances shown in Figures 1, 4, $91,92,56$ and 97 are computed directly from the tree generated via IQTree. Such distances were computed for every leaf of the tree (i.e. every sequence in our data set) and then averaged for sequences sampled in the same year for visualization purposes. Taking into account the phylogenetic information allows one to detect some mutations that would occur back and forth along the tree. Consequently, the estimates of the RTT distance are higher than the Hamming distance ones.

\subsection{Consensus and founder sequence}

Consensus sequences were computed from our BH data sets. We computed three consensus sequences for each region studied. First is the HIV-1 group M global consensus, which is the majority nucleotide of the alignment at each position. Second and third are the subtype B and subtype C consensus sequences. These were computed in the same way, using a subset of the alignment that contains only the sequences of the subtype in question.

The founder sequence is an approximation of the sequence of the virus at the time of infection in a patient. They are computed from our WH data set for each patient separately. The founder sequence is the majority nucleotide in each position from the first sample of each patient. In this sense, it is the consensus sequence obtained from the first sample of each patient. For most patients in our data set, the first sample is taken at approximately 90 days after infection and no data is available on the early phase of infection. Consequently, the founder sequence computed is an approximation of the original virus.

\subsection{Trajectory extraction and metadata}

A trajectory is a sequence of nucleotide frequencies and associated time. Each trajectory corresponds to one genome position and one nucleotide only. We extracted trajectories from our WH data set according to several criterion. Firstly, every trajectory is extinct before the first point, i.e. we consider only new mutations. This is to avoid biases that could be due to immune interaction existing already. Secondly, frequencies are between 0.01 and 0.99 at all time points. The trajectory is considered extinct if it is below 0.01 , and fixed if above 0.99. Lastly, we apply a mask to data points according to what is shown in section M\&M 1.1. Trajectories that have their first and/or last point masked are removed from the analysis.

Every trajectory extracted according to the criterion above is coupled with its metadata. This contains all the relevant information, such as whether the mutation is a reversion or not and whether it fixed or was lost. This information is used to create subgroups of trajectories. From these subgroups, one can study the impact of a trait associated with a mutation for WH evolution, as shown in Figure 3 for reversion and non-reversion trajectories.

\subsection{Mean frequency in time}

While looking at divergence values informs us on the global evolution of the WH population, it cannot tell us whether the mutations we see on non-consensus sites are actually reversions to the consensus state or simply mutations to another nucleotide. This motivated us to look directly at the evolution of new mutations independently by observing the frequency trajectories in time. Trajectories were extracted and 
filtered according to sections M\&M 1.1 and M\&M 1.4. Despite these filtering steps, our data is inherently biased towards small and/or low-frequency trajectories which are more common. In order to alleviate this bias, we compare reversion and non-reversion trajectories in the same manner. Accordingly, the resulting signal can be attributed to the effect of being a reversion (or not).

Due to the limited number of trajectories available and the often lack of information about trajectory fixation, for example, because it is still active at the last sample, the probability of fixation plots were not adequate for our analysis. We, thus, decided to pay attention to the evolution of the mean frequency in time of groups of trajectories. Trajectories were grouped in frequency bins, as described in the main text, to avoid bias towards positively or negatively selected trajectories.

We then created time bins of 400 days from 600 days before up to 3000 days after a trajectory is seen in a frequency window. We compute the average frequency of all trajectories belonging to the same group in each time bin. A trajectory contributes its current frequency if a data point is available at this time, and does not contribute if no data is available in that time bin. Trajectories that fixed in the population contribute with a frequency of $f=1$ to time bins subsequent to their fixation. Similarly, lost trajectories contribute $f=0$ to time bins subsequent to their disappearance in the viral population. Trajectories that are still active after their last data point (because the study stopped before it could fix or be lost) contribute the frequency of their last data point to the following time bins.

\section{Code and data availability}

The code and data used for the analysis can be found at https://github.com/neherlab/HIVEVO_reversion. Due to issues with the data sets' size, only intermediate $\mathrm{BH}$ and $\mathrm{WH}$ data files in a compressed format are found in the github folder. A link to the full data set is available there. Scripts are present to reproduce the results shown in this paper.

\section{References}

Aiewsakun Pakorn, Katzourakis Aris. Time-Dependent Rate Phenomenon in Viruses // Journal of Virology. VI 2016. Publisher: American Society for Microbiology 1752 N St., N.W., Washington, DC.

Alizon Samuel, Fraser Christophe. Within-host and between-host evolutionary rates across the HIV-1 genome // Retrovirology. 2013. 10, 1. 1-10.

Boutwell Christian L, Rolland Morgane M, Herbeck Joshua T, Mullins James I, Allen Todd M. Viral evolution and escape during acute HIV-1 infection // The Journal of infectious diseases. 2010. 202, Suppl 2. S309.

Brian Foley Cristian Apetrei et al Thomas Leitner. HIV Sequence Database: 2018 Compendium. 2018.

Carlson Jonathan M., Brumme Zabrina L. HIV evolution in response to HLA-restricted CTL selection pressures: a population-based perspective // Microbes and Infection. IV 2008. 10, 5. 455-461.

Carlson Jonathan M., Schaefer Malinda, Monaco Daniela C., Batorsky Rebecca, Claiborne Daniel T., Prince Jessica, Deymier Martin J., Ende Zachary S., Klatt Nichole R., DeZiel Charles E., Lin Tien-Ho, Peng Jian, Seese Aaron M., Shapiro Roger, Frater John, Ndung'u Thumbi, Tang Jianming, Goepfert Paul, Gilmour Jill, Price Matt A., Kilembe William, Heckerman David, Goulder Philip J. R., Allen Todd M., Allen Susan, Hunter Eric. HIV transmission. Selection bias at the heterosexual HIV-1 transmission bottleneck // Science (New York, N.Y.). VII 2014. 345, 6193. 1254031.

Coffin John, Swanstrom Ronald. HIV Pathogenesis: Dynamics and Genetics of Viral Populations and Infected Cells // Cold Spring Harbor Perspectives in Medicine. I 2013. 3, 1. a012526. Publisher: Cold Spring Harbor Laboratory Press.

Crawford Hayley, Prado Julia G, Leslie Alasdair, Hué Stéphane, Honeyborne Isobella, Reddy Sharon, Stok Mary van der, Mncube Zenele, Brander Christian, Rousseau Christine, others . Compensatory mutation partially restores fitness and delays reversion of escape mutation within the immunodominant HLA-B* 5703-restricted Gag epitope in chronic human immunodeficiency virus type 1 infection // Journal of virology. 2007. 81, 15. 8346-8351. 
Doud Michael B., Ashenberg Orr, Bloom Jesse D. Site-Specific Amino Acid Preferences Are Mostly Conserved in Two Closely Related Protein Homologs // Molecular Biology and Evolution. XI 2015. 32, 11. 2944-2960.

Felsenstein Joseph. Inferring phylogenies. 2004.

Foley B, Leitner T, Apetrei C, Hahn B, Mizrachi I, Mullins J, Rambaut A, Wolinsky S, Korber B. Theoretical Biology and Biophysics Group // Los Alamos: Los Alamos National Laboratory. 2013. 13.

Freed Eric O. HIV-1 replication // Somatic cell and molecular genetics. 2001. 26, 1. 13-33.

Ghafari Mahan, Simmonds Peter, Pybus Oliver G., Katzourakis Aris. Prisoner of War dynamics explains the time-dependent pattern of substitution rates in viruses // bioRxiv. II 2021. 2021.02.09.430479. Publisher: Cold Spring Harbor Laboratory Section: New Results.

Gilbert Clément, Feschotte Cédric. Genomic fossils calibrate the long-term evolution of hepadnaviruses // PLoS biology. IX 2010. 8, 9. e1000495.

Halpern A. L., Bruno W. J. Evolutionary distances for protein-coding sequences: modeling site-specific residue frequencies. // Molecular Biology and Evolution. VII 1998. 15, 7. 910-917.

Hanada Kousuke, Suzuki Yoshiyuki, Gojobori Takashi. A large variation in the rates of synonymous substitution for RNA viruses and its relationship to a diversity of viral infection and transmission modes // Molecular Biology and Evolution. VI 2004. 21, 6. 1074-1080.

Hilton Sarah K., Bloom Jesse D. Modeling site-specific amino-acid preferences deepens phylogenetic estimates of viral sequence divergence // Virus Evolution. VII 2018. 4, 2.

Ho Simon Y. W., Phillips Matthew J., Cooper Alan, Drummond Alexei J. Time Dependency of Molecular Rate Estimates and Systematic Overestimation of Recent Divergence Times // Molecular Biology and Evolution. VII 2005. 22, 7. 1561-1568.

Huddleston John, Hadfield James, Sibley Thomas R., Lee Jover, Fay Kairsten, Ilcisin Misja, Harkins Elias, Bedford Trevor, Neher Richard A., Hodcroft Emma B. Augur: a bioinformatics toolkit for phylogenetic analyses of human pathogens // Journal of Open Source Software. I 2021. 6, 57. 2906.

Kalyaanamoorthy Subha, Minh Bui Quang, Wong Thomas KF, Von Haeseler Arndt, Jermiin Lars S. ModelFinder: fast model selection for accurate phylogenetic estimates // Nature methods. 2017. 14, 6. 587-589.

Katoh Kazutaka, Standley Daron M. MAFFT Multiple Sequence Alignment Software Version 7: Improvements in Performance and Usability // Molecular Biology and Evolution. IV 2013. 30, 4. 772-780.

Korber Bette, Muldoon Mark, Theiler James, Gao Fei, Gupta Radhika, Lapedes Alan, Hahn BH, Wolinsky Steven, Bhattacharya Tanmoy. Timing the ancestor of the HIV-1 pandemic strains // science. 2000. 288, 5472. 1789-1796.

Leslie AJ, Pfafferott KJ, Chetty P, Draenert R, Addo MM, Feeney M, Tang Y, Holmes EC, Allen T, Prado $J G$, others. HIV evolution: CTL escape mutation and reversion after transmission // Nature medicine. 2004. 10, 3. 282-289.

Li Guangdi, Piampongsant Supinya, Faria Nuno Rodrigues, Voet Arnout, Pineda-Peña Andrea-Clemencia, Khouri Ricardo, Lemey Philippe, Vandamme Anne-Mieke, Theys Kristof. An integrated map of HIV genome-wide variation from a population perspective // Retrovirology. 2015. 12, 1. 18.

Lythgoe Katrina A, Fraser Christophe. New insights into the evolutionary rate of HIV-1 at the within-host and epidemiological levels // Proceedings of the Royal Society B: Biological Sciences. 2012. 279, 1741. $3367-3375$.

McCutchan Francine E. Global epidemiology of HIV // Journal of medical virology. 2006. 78, S1. S7-S12.

Minh Bui Quang, Schmidt Heiko A, Chernomor Olga, Schrempf Dominik, Woodhams Michael D, Von Haeseler Arndt, Lanfear Robert. IQ-TREE 2: new models and efficient methods for phylogenetic inference in the genomic era // Molecular biology and evolution. 2020. 37, 5. 1530-1534. 
Puller Vadim, Sagulenko Pavel, Neher Richard A. Efficient inference, potential, and limitations of site-specific substitution models // Virus Evolution. VII 2020. 6, 2.

Raghwani Jayna, Redd Andrew D., Longosz Andrew F., Wu Chieh-Hsi, Serwadda David, Martens Craig, Kagaayi Joseph, Sewankambo Nelson, Porcella Stephen F., Grabowski Mary K., Quinn Thomas C., Eller Michael A., Eller Leigh Anne, Wabwire-Mangen Fred, Robb Merlin L., Fraser Christophe, Lythgoe Katrina A. Evolution of HIV-1 within untreated individuals and at the population scale in Uganda // PLOS Pathogens. VII 2018. 14, 7. e1007167. Publisher: Public Library of Science.

Redd Andrew D., Collinson-Streng Aleisha N., Chatziandreou Nikolaos, Mullis Caroline E., Laeyendecker Oliver, Martens Craig, Ricklefs Stacy, Kiwanuka Noah, Nyein Phyu Hninn, Lutalo Tom, Grabowski Mary K., Kong Xiangrong, Manucci Jordyn, Sewankambo Nelson, Wawer Maria J., Gray Ronald H., Porcella Stephen F., Fauci Anthony S., Sagar Manish, Serwadda David, Quinn Thomas C. Previously Transmitted HIV-1 Strains Are Preferentially Selected During Subsequent Sexual Transmissions // The Journal of Infectious Diseases. XI 2012. 206, 9. 1433-1442.

Sagulenko Pavel, Puller Vadim, Neher Richard A. TreeTime: Maximum-likelihood phylodynamic analysis // Virus Evolution. 01 2018. 4, 1. vex042.

Sharp Paul M, Hahn Beatrice H. Origins of HIV and the AIDS pandemic // Cold Spring Harbor perspectives in medicine. 2011. 1, 1. a006841.

Soubrier Julien, Steel Mike, Lee Michael SY, Der Sarkissian Clio, Guindon Stéphane, Ho Simon YW, Cooper Alan. The influence of rate heterogeneity among sites on the time dependence of molecular rates // Molecular biology and evolution. 2012. 29, 11. 3345-3358.

Tavaré Simon, others . Some probabilistic and statistical problems in the analysis of DNA sequences // Lectures on mathematics in the life sciences. 1986. 17, 2. 57-86.

Wertheim Joel O., Kosakovsky Pond Sergei L. Purifying Selection Can Obscure the Ancient Age of Viral Lineages // Molecular Biology and Evolution. XII 2011. 28, 12. 3355-3365.

Worobey Michael, Telfer Paul, Souquière Sandrine, Hunter Meredith, Coleman Clint A., Metzger Michael J., Reed Patricia, Makuwa Maria, Hearn Gail, Honarvar Shaya, Roques Pierre, Apetrei Cristian, Kazanji Mirdad, Marx Preston A. Island Biogeography Reveals the Deep History of SIV // Science. IX 2010. 329, 5998. 1487-1487. Publisher: American Association for the Advancement of Science Section: Brevia.

Yang Ziheng. A space-time process model for the evolution of DNA sequences. // Genetics. 1995. 139, 2. 993-1005.

Zanini Fabio, Brodin Johanna, Albert Jan, Neher Richard A. Error rates, PCR recombination, and sampling depth in HIV-1 Whole Genome Deep Sequencing. // Virus research. December 2016.

Zanini Fabio, Brodin Johanna, Thebo Lina, Lanz Christa, Bratt Göran, Albert Jan, Neher Richard A. Population genomics of intrapatient HIV-1 evolution // Elife. 2015. 4. e11282.

Zanini Fabio, Neher Richard A. Quantifying Selection against Synonymous Mutations in HIV-1 env Evolution // Journal of Virology. XI 2013. Publisher: American Society for Microbiology 1752 N St., N.W., Washington, DC.

Zanini Fabio, Puller Vadim, Brodin Johanna, Albert Jan, Neher Richard A. In vivo mutation rates and the landscape of fitness costs of HIV-1 // Virus evolution. 2017. 3, 1. 


\section{Supplementary Materials}
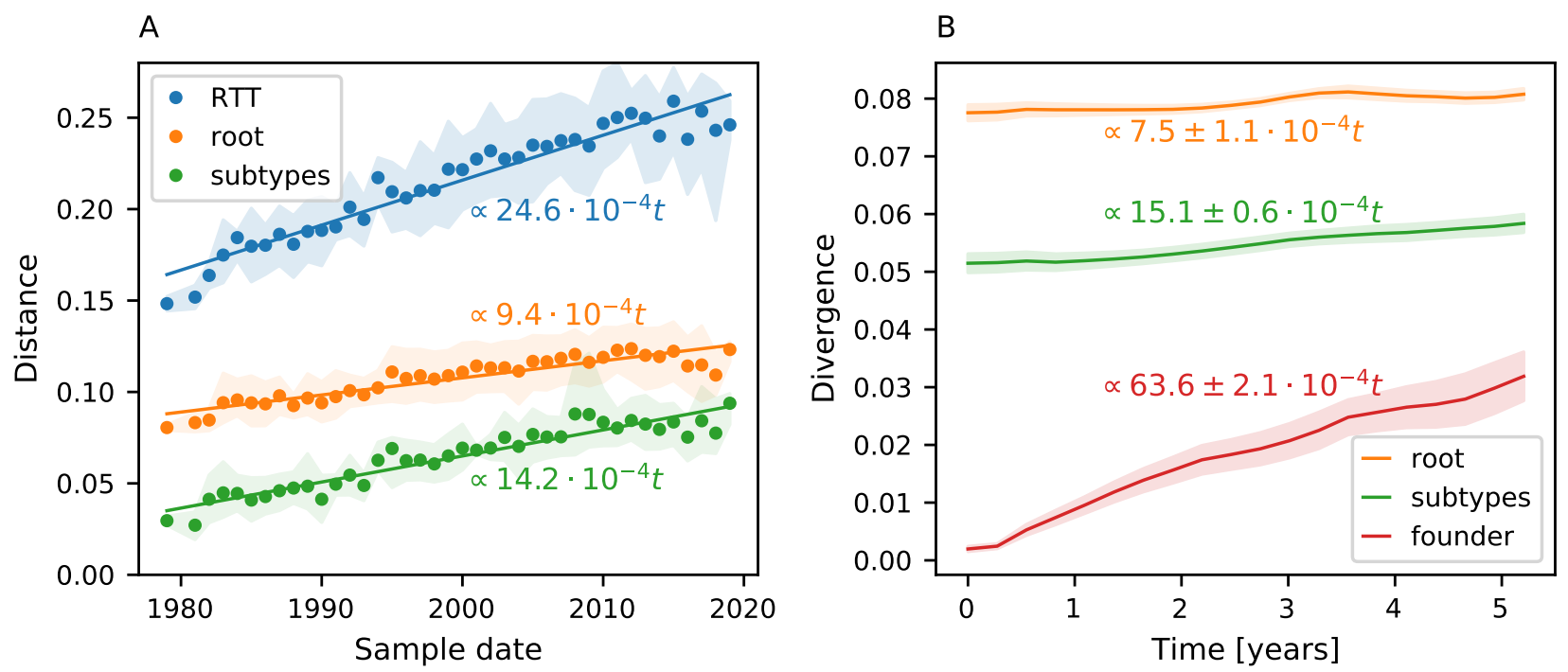

Supp. Fig. S1: Same as Figure 1 for the env gene. The Y axes are not shared in this case. The env gene behaves similarly to pol and gag but the difference in the rate observed is higher. This is consistent with the fact that env mutates faster overall, which would also lead to more reversions. 

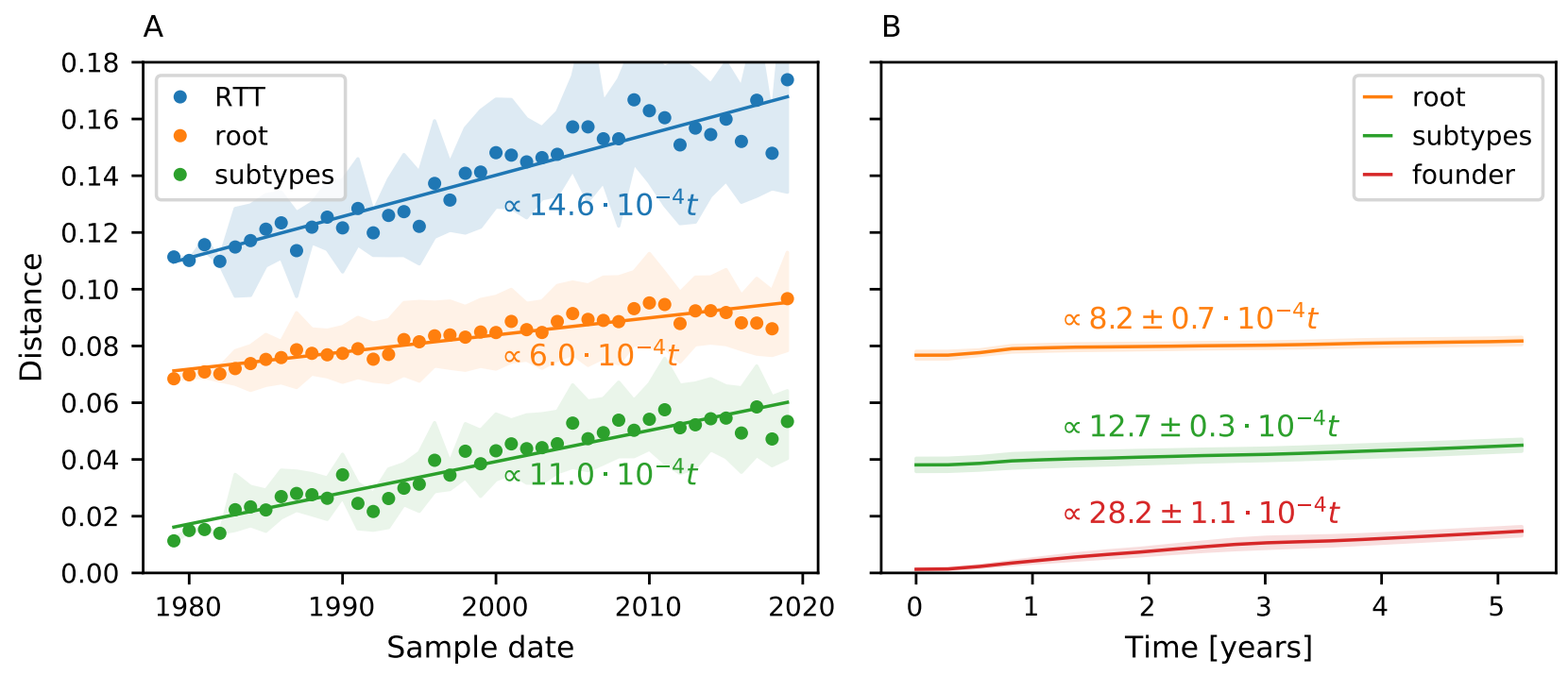

Supp. Fig. S2: Same as Figure 1 for the gag gene.
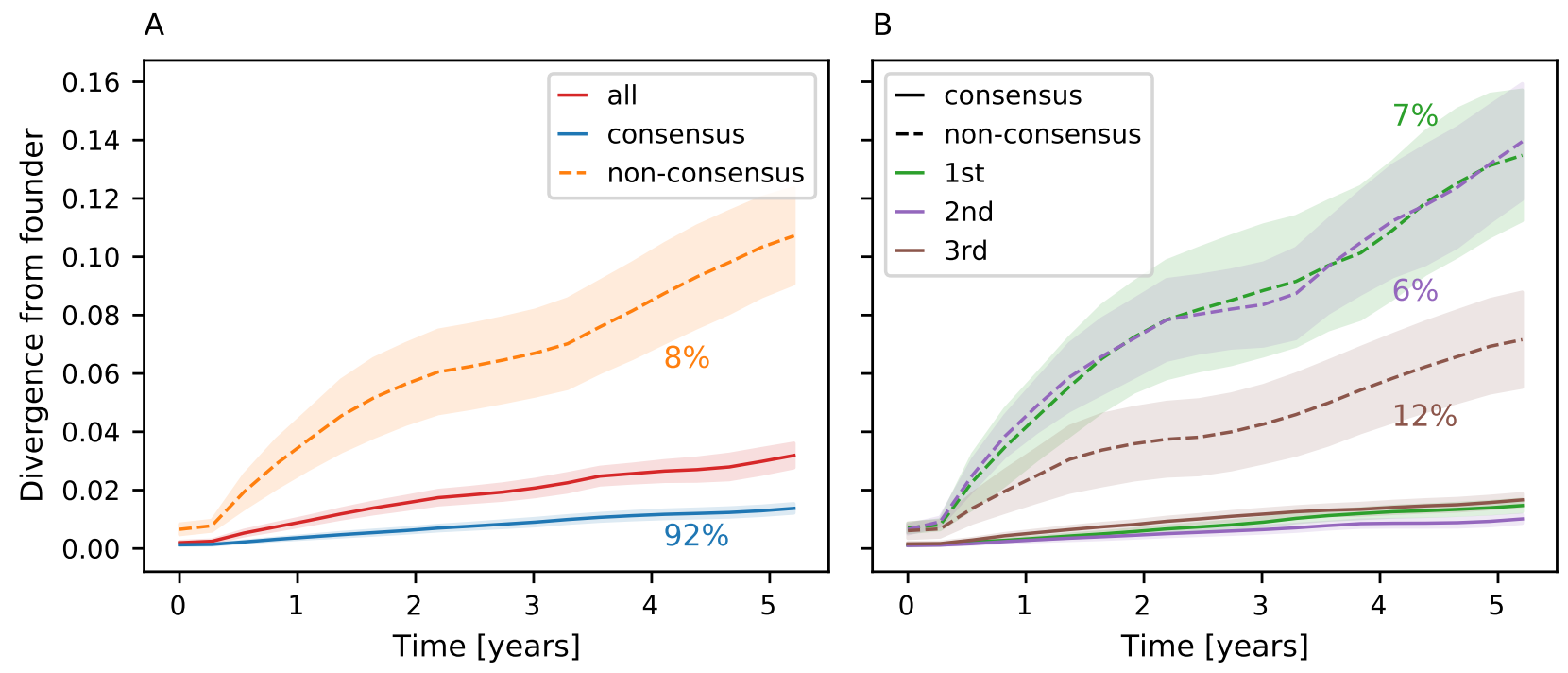

Supp. Fig. S3: Same as Figure 2 for the env gene. 

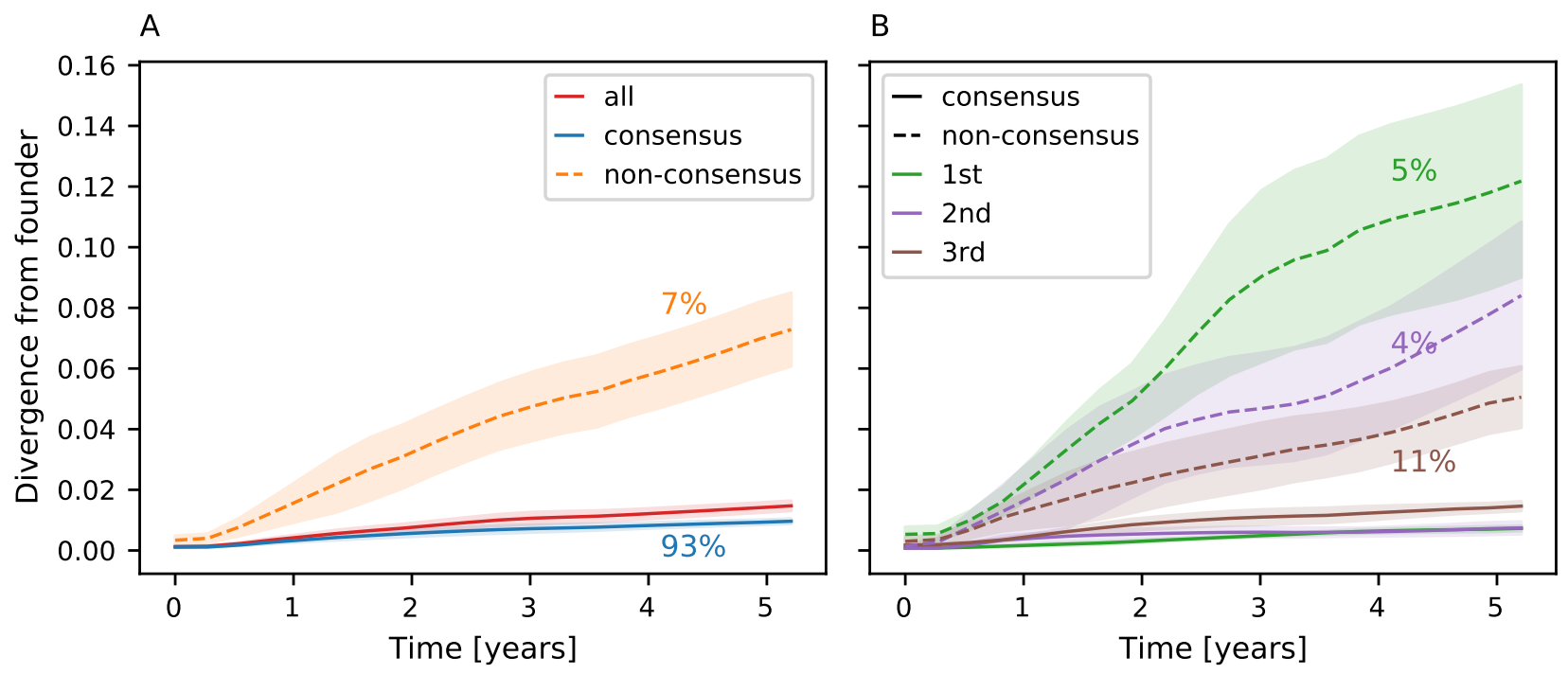

Supp. Fig. S4: Same as Figure 2 for the gag gene.
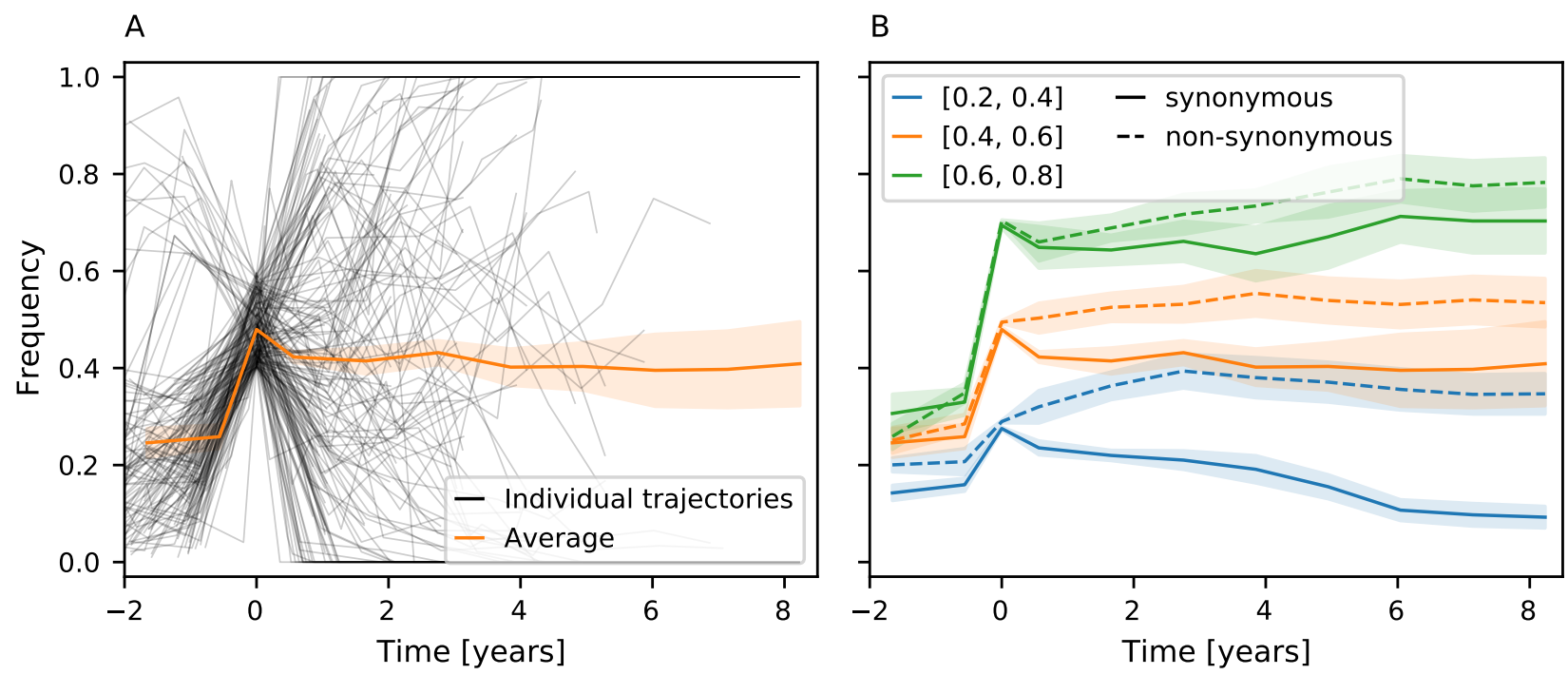

Supp. Fig. S5: Same as Figure 3 but for synonymous and non-synonymous trajectories. Overall synonymous mutations are selected against and non-synonymous mutations seem to be selected for, but the effect is smaller than what we see for reversions and non-reversions in Figure 3. 

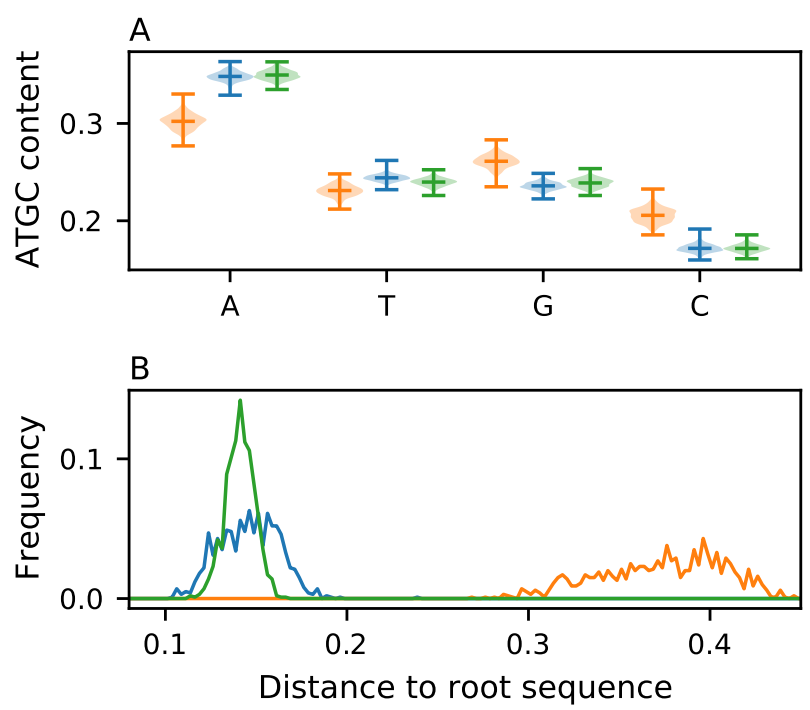

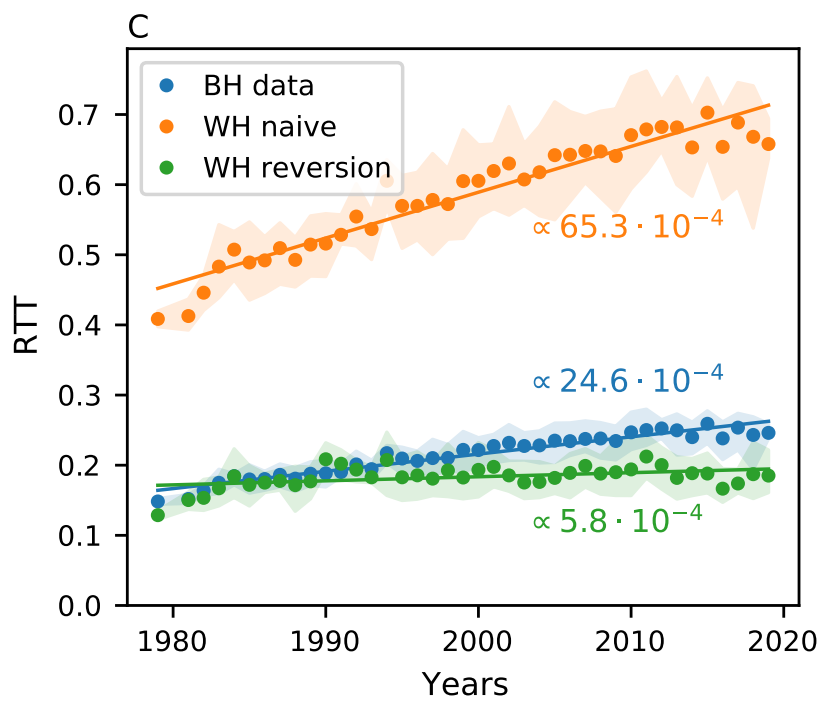

Supp. Fig. S 6: Same as Figure 4 for the env gene. The WH mutation rate in this region is so high that the reversion model attenuates most of the clock signal, which leads the tree reconstruction to fail.
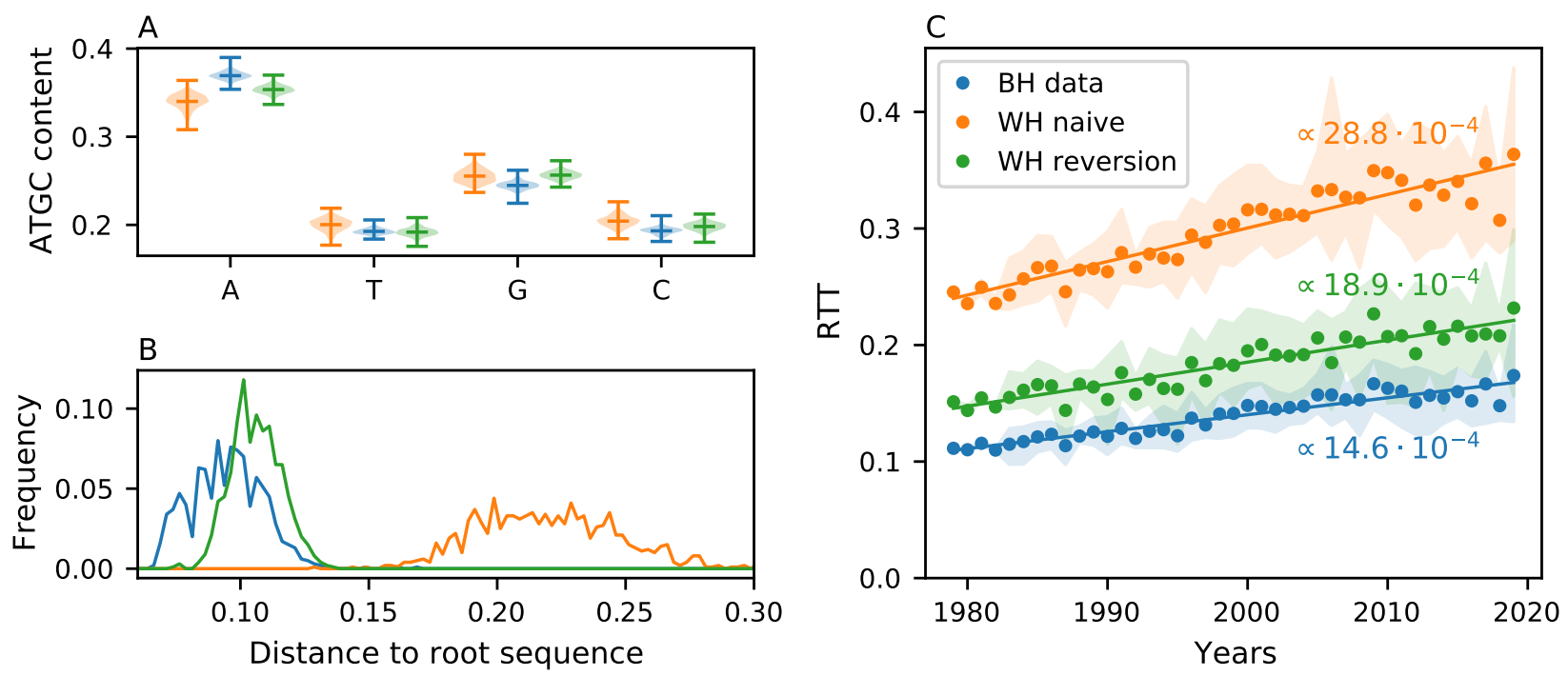

Supp. Fig. S7: Same as Figure 4 for the gag gene. 

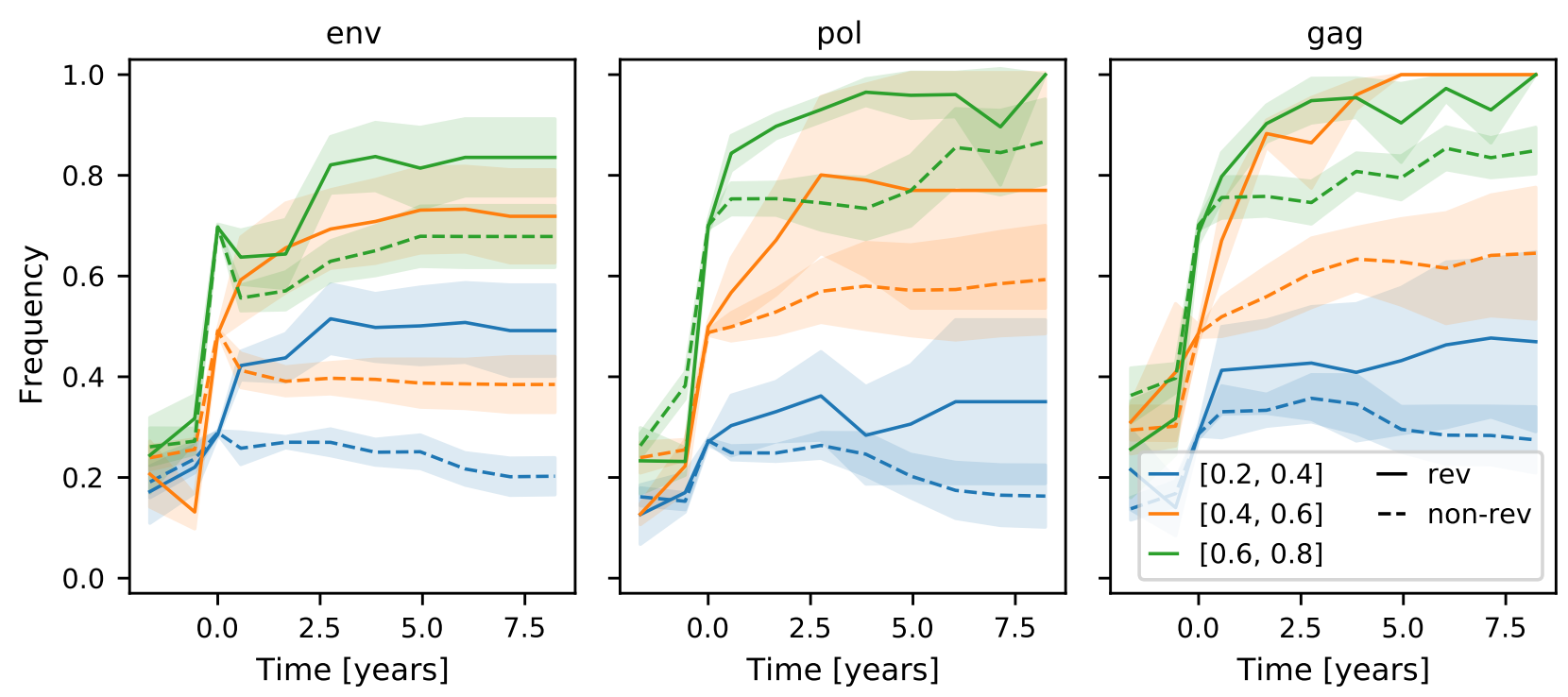

Supp. Fig. S 8: Same as Figure 3 B split per region. Selection for reversion is strongest in the gag region and weakest in the $e n v$ region. 